EUROPEAN JOURNAL OF PURE AND APPLIED MATHEMATICS

Vol. 12, No. 2, 2019, 649-653

ISSN 1307-5543 - www.ejpam.com

Published by New York Business Global

\title{
Some estimates below the modulus of integrals of some polynomials in the complex plane
}

Todor Stoyanov Stoyanov

1 University of Economics, Department of Mathematics bul. Knyaz Boris I r7, Varna 9002, Bulgaria

\begin{abstract}
In this paper, we make some estimates below the modulus of some integrals in the complex plane. Our aim is to prove the Conjecture1, which we could see in [2-4]. The proof of the conjecture appears the Corollary.
\end{abstract}

2010 Mathematics Subject Classifications: 30A10

Key Words and Phrases: Zeros, complex polynomial, real polynomial, disk, derivative, integral

\section{Introduction}

In papers [2-4], we consider the Conjecture 1: If $a_{k} \geq 0, a_{k} \in \mathbb{R}$, Then we assert

$$
\left|\int_{0}^{e^{i \varphi}} \prod_{k=1}^{n}\left(x+a_{k}\right) d x\right| \geq \frac{1}{n+1},
$$

for arbitrary natural $n, \varphi \in\left[0, \frac{\pi}{2}\right]$. There exists a connection between this conjecture and Conjecture2: If $\phi_{k} \in\left[\frac{\pi}{2}, \pi\right]$, then

$$
\left|\int_{-1}^{0}(x+1) \prod_{k=1}^{n}\left(x-e^{i \phi_{k}}\right) d x\right| \geq \frac{1}{n+2} .
$$

Both conjectures are very important for the proofs of some famous conjectures, like Sendov's and Obreshkoff's ones. A possible connection between both conjctures appears [5]. Here we shall extend this problem (Conjecture1): what kind of set $L$ satisfies this assertion, i.e. if $a_{k}$ belongs to the set $L$, then the upper inequality is true. The results related with the Conjecture 1, we observe in Theorem 1 , Theorem 2. In Theorem 4 we generalize and prove the extended conjecture. We can see the results of Theorem 1 in

DOI: https://doi.org/10.29020/nybg.ejpam.v12i2.3372

Email address: todstoyanov@yahoo.com (T. S. Stoyanov) 
$[2,4]$. Such one of Theorem 2 could be seen in [3]. Many authors use some modulus of some integrals in the complex plane for various estimates in their works. For example we can see how Bojanov and Rahman in [1] use this method. These estimates are explored for the localization of the zeros of some polynomials. The results are useful in the (open) problems of $[6-9]$.

\section{Related Results}

Theorem 1. Let $k=1,2, \ldots n, n \in \mathbb{N}, a_{k} \in[0,1] \varphi \in\left[0, \frac{\pi}{2}\right]$. Then the function

$$
\left|\int_{-1}^{e^{i \varphi}} x \prod_{k=1}^{n}\left(x+a_{k}\right) d x\right| \geq \frac{1}{n+2}
$$

for $n=1,2,3$.

Theorem 2. let $k \in \mathbb{N}, a \in \mathbb{R}, a \in[0,1]$. Then the function

$$
\left|\int_{0}^{i} x \quad(x+a)^{k} d x\right| \geq \frac{1}{n+2} .
$$

\section{Preliminaries}

We note:

$D(a, r)=\{z \in \mathbb{C}:|z-a|<r\}$ is the open disk with center $a$ and radius $r$.

$\bar{D}(0, r)=\{z \in \mathbb{C}:|z-a| \leq r\}$ is the closed disk with center $a$ and radius $r$.

$A=\{z \in \mathbb{C}, \operatorname{Re} z \leq 0\}$ is the left semiplane.

\section{Main Results}

Theorem 3. We consider a polynomial $r(z)=z^{n-1}+r_{n-1} z^{n-1}+\ldots+r_{1} z+r_{0}$. where $r_{k} \in \mathbb{R}, n \geq 1, n \in \mathbb{N}, k=\overline{0, n-1}$. The zeros $z_{k}$ of $r(z)$ satisfy the condition Re $z_{k} \leq 0$. If $a \geq 0$, then $I=n \int_{0}^{a} r(z) d z \geq a^{n}$.

Proof. Let $r(z)=\left(z+a_{1}\right)\left(z+a_{2}\right) \ldots\left(z+a_{1}\right)\left(z-b_{1}\right)\left(z-\overline{b_{1}}\right) \ldots\left(z-b_{s}\right)\left(z-\overline{b_{s}}\right)$, where $1+2 s=n-1, a_{k} \geq 0, b_{m} \in \mathbb{C}, k=\overline{1, l}, m=\overline{1, s}, a_{k} \in \mathbb{R}, k, m \in \mathbb{N}$. and $b_{m}=\rho_{m} e^{i \varphi m}, \rho_{m} \geq$ $0, \varphi_{m} \in\left[\frac{\pi}{2}, \pi\right],\left(z-b_{m}\right)\left(z-\overline{b_{m}}\right)=z^{2}-2 \rho_{m} \cos \varphi_{m} z+\rho_{m}^{2} \geq z^{2}$. Then $\begin{aligned} n \int_{0}^{a} r(z) d z & =n \int_{0}^{a}\left(z+a_{1}\right)\left(z+a_{2}\right) \ldots\left(z+a_{1}\right)\left(z-b_{1}\right)\left(z-\overline{b_{1}}\right) \ldots\left(z-b_{s}\right)\left(z-\overline{b_{s}}\right) d \geq \\ n \int_{0}^{a} z^{n-1} d z & =a^{n} .\end{aligned}$ 
Theorem 4. We consider a polynomial $r(z)=z^{n-1}+r_{n-1} z^{n-2}+\ldots+r_{1} z+r_{0}$, where $r_{k} \in \mathbb{R}, n \geq 1, n \in \mathbb{N}, k=\overline{0, n-1}$. The zeros $z_{k}$ of $r(z)$ satisfy the condition $z_{k} \in$ $A \backslash D\left(z_{0}, a\right) \backslash D\left(\overline{z_{0}}, a\right), z_{0}=a e^{i \theta_{0}}$, where $a \geq 0, \theta_{0} \in\left[0, \frac{\pi}{2}\right]$. Then

$$
I=\left|n \int_{0}^{z_{0}} r(z) d z\right| \geq a^{n} .
$$

Proof. Let us put $v(\theta)=a e^{i \theta}, \theta \in\left[0, \theta_{0}\right], I+2 s=n-1$,

$$
r(z)=\prod_{p=1}^{l}\left(z+a_{p}\right) \prod_{p=1}^{s}\left(z-b_{p}\right)\left(z-\overline{b_{p}}\right),
$$

$l, s \in \mathbb{N}$ (one of the factors could be not existing, i.e., $l=0$ or $s=0$ ).

We put $f(\theta)=n \int_{0}^{v(\theta)} r(z) d z, g(\theta)=f(\theta) \cdot \bar{f}(\theta)$.

Let us calculate

$$
\begin{gathered}
\frac{d g}{d \theta}=n\left[r(v(\theta)) \frac{d v}{d \theta} \bar{f}(\theta)+r(\bar{v}(\theta)) \frac{d \bar{v}}{d \theta} f(\theta)\right], \\
\frac{d v}{d \theta}=\frac{d a e^{i \theta}}{d \theta}=i a e^{i \theta}
\end{gathered}
$$

and if we put

$$
\begin{gathered}
U_{0}=v(\theta)=a e^{i \theta}, U_{p}=v(\theta)+a_{p}, p=\overline{1, l}, U_{l+2 p+1}=v(\theta)-b_{p}, \\
U_{l+2 p+2}=v(\theta)-\overline{b_{p}}, p=\overline{0, s-1 .}
\end{gathered}
$$

Knowing

$$
\frac{d f}{d \theta} \cdot \Pi_{p=0}^{n-1} \overline{U_{p}}=\frac{d \bar{f}}{d \theta} \cdot \Pi_{p=0}^{n-1} U_{p}
$$

we have

$$
\begin{gathered}
\frac{d g}{d \theta}=i n\left[\bar{f}(\theta) \prod_{p=0}^{n-1} U_{p}-f(\theta) \prod_{p=0}^{n-1} \overline{U_{p}}\right], \\
\frac{d^{2} g}{d \theta^{2}}=n\left[2 \frac{d f}{d \theta} \prod_{p=0}^{n-1} \overline{U_{p}}+i \frac{d \prod_{p=0}^{n-1} U_{p}}{d \theta} \bar{f}(\theta)-i \frac{d \Pi_{p=0}^{n-1} \overline{U_{p}}}{d \theta} f(\theta)\right], \\
\frac{d^{2} g}{d \theta^{2}}=n\left[2 n \prod_{p=0}^{n-1}\left|U_{p}\right|^{2}-\left(U_{0} \sum_{p=0}^{n-1} \prod_{j \neq p} U_{j}\right) \bar{f}(\theta)-\left(\overline{U_{0}} \sum_{p=0}^{n-1} \prod_{j \neq p} \overline{U_{j}}\right) f(\theta)\right], \\
\frac{d^{2} g}{d \theta^{2}}=2 n\left[n \prod_{p=0}^{n-1}\left|U_{p}\right|^{2}-\operatorname{Re}\left(U_{0} \sum_{p=0}^{n-1} \prod_{j \neq p} U_{j}\right) \bar{f}(\theta)\right],
\end{gathered}
$$


and consequently

$$
\frac{d^{2} g}{d \theta^{2}} \geq 2 n \prod_{p=0}^{n-1}\left|U_{p}\right|\left[n \prod_{p=0}^{n-1}\left|U_{p}\right|-\frac{\left|U_{0} \sum_{p=0}^{n-1} \prod_{j \neq p} U_{j}\right| \cdot|\bar{f}(\theta)|}{\Pi_{p=0}^{n-1} U_{p}}\right] .
$$

If we note

$$
B=\{A \backslash D(z, a) \backslash D(\bar{z}, a)\}, B_{0}=\left\{A \backslash D\left(z_{0}, a\right) \backslash D\left(\overline{z_{0}}, a\right)\right\}
$$

and since

$$
\theta \in\left[0, \theta_{0}\right] \Longrightarrow B_{0} \subset B, \text { i.e., }\left|U_{p}(\theta)\right| \geq a, p=\overline{1, n-1} .
$$

If we assume

$$
|f(\theta)|=|\bar{f}(\theta)| \leq a^{n}
$$

then

$$
\begin{gathered}
\frac{d^{2} g}{d \theta^{2}} \geq 2 n \prod_{p=0}^{n-1}\left|U_{p}\right|\left[n a a^{n-1}-\left(1+\left|\frac{U_{0}}{U_{1}}\right|+\ldots+\left|\frac{U_{0}}{U_{n-1}}\right|\right) \cdot a^{n}\right] \\
\geq 2 n a \cdot \Pi_{p=0}^{n-1}\left|U_{p}\right|\left[n a^{n-1}-\left(1+\frac{a(n-1)}{a}\right) \cdot a^{n-1}\right]=0 .
\end{gathered}
$$

Then

$$
\frac{d^{2} g}{d \theta^{2}} \geq 0
$$

Hence

$$
\frac{d g}{d \theta}(\theta) \geq \frac{d g}{d \theta}(0)=0 .
$$

Consequently $g\left(\theta_{0}\right)>g(0)$, i.e., $\left|f\left(\theta_{0}\right)\right|>a^{n}$, according to the proof of Theorem 3 . Therefore $a^{n}<\left|f\left(\theta_{0}\right)\right| \leq a^{n}$, which is impossible. The contradiction proves the Theorem 4.

Corollary. If in the condition of Theorem 4 , we put $a=1$, and $s=0$, i.e., all the zeros of $\mathrm{r}(z)$ are real and negative, then we get that the Conjecture 1 is true.

\section{Acknowledgements}

This research was financed from University of Economics of Varna research grants No. 19 2018-04-27 


\section{References}

[1] Q.I. Rahman B. Bojanov and J. Szynal. On a conjecture of sendov about the critical points of a polynomial. Math.Z, 190:281-285, 1985.

[2] T. Stoyanov. Inequalities of the modulus of some complex integrals, theory and practice of modern science.

[3] T. Stoyanov. Some estimates below the modulus of integrals in the complex plane. International scientific conference, Tendencies and prospects of development of modern scientific knowledge, $\mathrm{V}$.

[4] T. Stoyanov. Some estimates of the modulus of some complex integrals using division of polynomials. International scientific conference, Tendencies and prospects of development of modern scientific knowledge, Moskwa, IV.

[5] T. Stoyanov. Some new methods for the estimates of the modulus of some integrals of the unit circle in the complex plane. Journal of Analysis and Applications, 17(2):119$130,2019$.

[6] T. Zapryanova. Approximation by the operators of cao-gonska type g+s,n. direct and converse theorem. Mathematics and Education in Mathematics.

[7] T. Zapryanova. Approximation by the operators of cao-gonska type gs,n and $\mathrm{g}^{*} \mathrm{~s}, \mathrm{n}$. direct and converse theorem. Mathematics and Education in Mathematics, pages 189$194,2008$.

[8] T. Zapryanova. A characterization of the k-functional for the algebraic version of the trigonometric jackson integrals gs,n and the k-functionals for cao-gonska operators g*s,n and g+s,n. Result. Math., 54:397-413, 2009.

[9] T. Zapryanova and D. Souroujon. On the iterates of jackson type operator gs,n. Mediterr. J. Math. 